\title{
Optoelectronic properties of poly(fluorene) co-polymer light-emitting devices on a plastic substrate*
}

\author{
Jerzy Kanicki \\ Shu-Jen Lee \\ Yongtaek Hong \\ Chia-Chen Su
}

\begin{abstract}
The optoelectronic properties of red, green, and blue poly(fluorene) co-polymer lightemitting devices (PLEDs) on a plastic substrate having a multi-layered structure with water vapor and oxygen transmission rates of less than $10^{-5} \mathrm{~g} / \mathrm{cm}^{2}$-day-atm and $10^{-7} \mathrm{cc} / \mathrm{cm}^{2}$-day-atm, respectively, is reported. A semitransparent thin metal multi-layer (i.e., $\mathrm{Au} / \mathrm{Ag} / \mathrm{Au}$ or $\mathrm{Ag} / \mathrm{Au} / \mathrm{Ag}$ ) is placed between the plastic substrate and the ITO coating, achieving a low sheet resistance of $12-13 \Omega / \square$ and an adequate optical transmission greater than $75 \%$. A wider color gamut and a maximum emission efficiency of $0.7,10$, and $1.7 \mathrm{~cd} / \mathrm{A}$ for red, green, and blue PLEDs, respectively, was obtained. Finally, a simple equivalent-circuit model was used to simulate the current-density-voltage characteristics of PLEDs.
\end{abstract}

Keywords - PLED, flexible plastic substrate, device efficiency, circuit model.

\section{Introduction}

Today, active-matrix organic light-emitting display (AMOLED) technology is considered as the next-generation flat-paneldisplay (FPD) technology for a large number of applications requiring different sized displays. Thus far, most of the organic light-emitting device (OLED) displays are fabricated on rigid glass ${ }^{1-4}$ or silicon substrates, ${ }^{5}$ and several groups have reported displays fabricated on flexible plastic substrates. ${ }^{6-9}$ The use of flexible substrates is important not only for making foldable displays possible, but also for reducing the thickness and weight and providing the potential of low-cost manufacturing of large-area FPDs. However, for such applications, the substrate materials must be available in high volume at low cost. Metal foils, metal-coated plastic foils, and thin flexible glass are possible candidates, as well as plastic materials. ${ }^{10}$ The initial investigation of flexible substrates for OLEDs was focused upon poly(ethylene terephthalate) (PET), ${ }^{11}$ polyester, ${ }^{12}$ and polyimide ${ }^{13}$ because of their low-cost potential and good optical properties, which are critical when the light is emitted through a plastic substrate. In addition to the desirable optical, surface, and electrical properties, ${ }^{14}$ it is important that the permeation of external oxygen and water through plastic substrate is minimized $^{10,15}$ by thermal out-gassing and additional coatings. The use of plastic substrate creates special problems in active-matrix displays. The standard thin-film-transistor (TFT) fabrication method, using either amorphous or polycrystalline silicon, requires temperatures above those that can be tolerated by most plastic substrates. ${ }^{9}$ In addition, the coefficient of thermal expansion (CTE) of plastic is usually much higher than that of silicon, so that heating leads to considerable mechanical stress. There problems are par- ticularly severe for poly-Si TFTs. It is not easy to find a plastic substrate that possesses all the optical, surface, electrical, chemical, and mechanical properties that are desirable not only for OLEDs but also for AMOLEDs. One of the possible candidates is the plastic substrate extensively used by our group over last several years. ${ }^{16-18}$ In this paper, we describe the organic polymer light-emitting devices (PLEDs) fabricated over the poly(dicylo-pentadiene) substrate, such as the material produced by LOFO High Tech Film GmbH under the trade name "Transphan." 19,20

\section{Plastic substrate properties}

The plastic substrate used for PLEDs has a multi-layered structure that includes the base film of polydicylo-pentadiene condensate, such as fabricated "transphan" which has a high glass transition temperature $\left(T_{g} \sim 170^{\circ} \mathrm{C}\right)$ and low birefringence [Fig. 1(a)]. LOFO casts "transphan og" from a solution of Arton $\mathrm{G}$ in methylene chloride; its chemical formula is given in Ref. 1. To enhance the substrate's thermal stability, optical characteristics, and gas-blocking property, a multi-layered oxygen/moisture barrier (for example, a- $\mathrm{SiO}_{x} /$ acrylic/a- $\mathrm{SiO}_{x}$ ) was deposited on top of the base film. The acrylic and low-temperature amorphous-silicon oxide $\left(\mathrm{a}-\mathrm{SiO}_{x}\right)$ can be used as a hard coat and oxygen/moisture barrier, respectively. ${ }^{19,20}$ To further reduce the gas transmission through the substrate, we added additional PECVD layers, such as a- $\mathrm{SiO}_{x}: \mathrm{H}$ and $\mathrm{a}-\mathrm{SiN}_{x}: \mathrm{H}$, on one side of the plastic substrate.

On top of the multi-layered substrate, the transparent conducting electrode (TCE-ITO) was defined either by dry etching using a laser-based method ${ }^{19}$ or by wet etching. ${ }^{20}$

\footnotetext{
*Review paper.

J. Kanicki is with the Solid-State Electronics Laboratory, Department of Electrical Engineering and Computer Science, and the Macromolecular Science \& Engineering Center, University of Michigan, Ann Arbor, MI, 48109 U.S.A.; telephone 734/936-0972, fax 734/615/2843, e-mail: kanicki@eecs.umich.edu.

S-J. Lee is with the Macromolecular Science \& Engineering Center, University of Michigan, Ann Arbor, MI, U.S.A.

Y. Hong and C-C. Su are with the Solid-State Electronics Laboratory, Department of Electrical Engineering and Computer Science, University of Michigan, Ann Arbor, MI, U.S.A.

(c) Copyright 2005 Society for Information Display 1071-0922/05/1312-0993\$1.00
} 


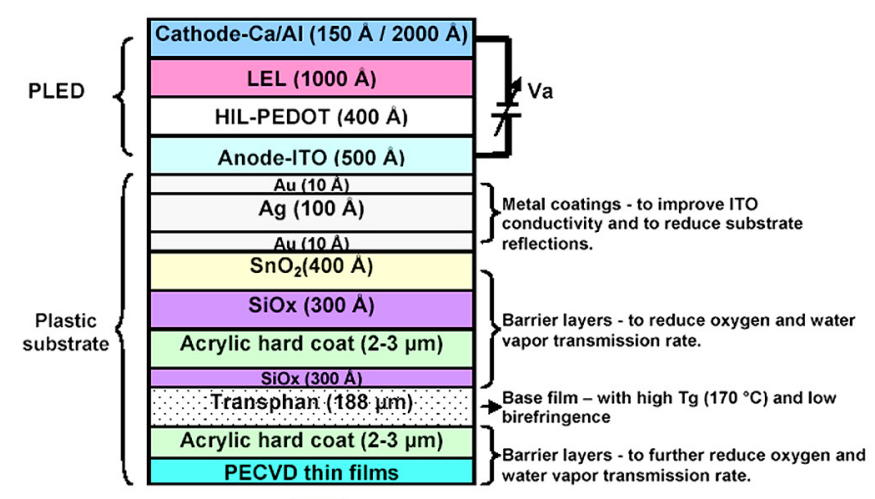

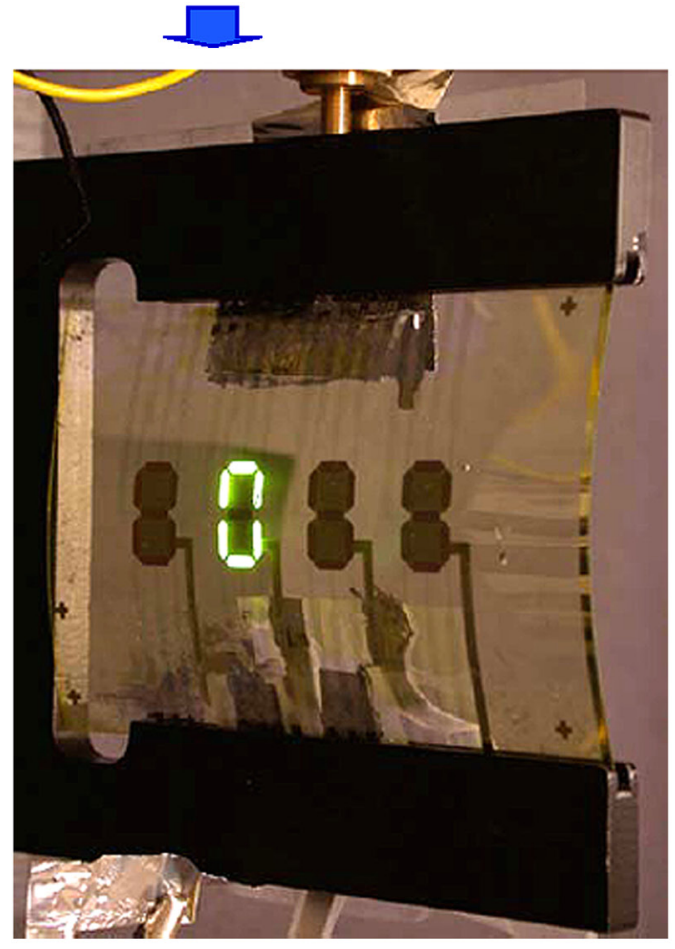

(a)

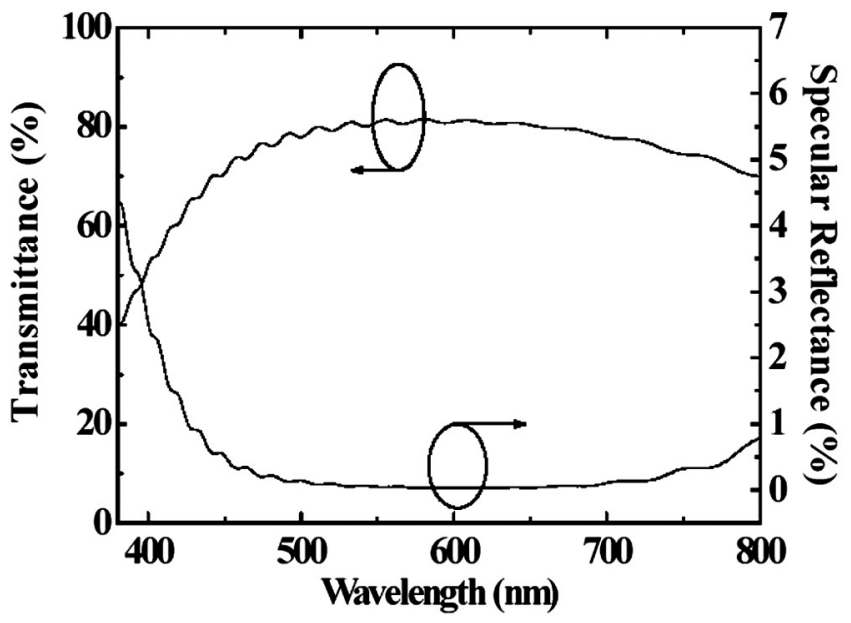

(b)

FIGURE 1 - (a) Schematic cross-sectional view and front view of a PLED fabricated on a plastic substrate. (b) Transmittance and specular reflectance spectra (measured at an incident angle of $7^{\circ}$ ) of the dry etchable plastic substrate.

To improve TCE conductivity without significantly affecting the optical transmission through the substrate, a semitransparent thin metal multi-layer (for example, $\mathrm{Au} / \mathrm{Ag} / \mathrm{Au}$ or $\mathrm{Ag} / \mathrm{Au} / \mathrm{Ag}$ ) is added between the indium tin oxide (ITO) and metal oxide (ITO or $\mathrm{SnO}_{2}$ ) layers. It is well known that a very thin silver or silver containing palladium layer placed between transparent conducting oxide layers allows for a very high electric conductivity, a good mechanical durability, and a high transparency in the visible range due to the anti-reflection effect. ${ }^{21}$ Our TCE coating on this plastic substrate has a sheet resistance of $12-13 \Omega / \square$, an optical transmittance of greater than $75 \%$ and a specular reflectance of less than $3 \%$ over the visible range [Fig. 1(b)], and a rootmean-square (RMS) surface roughness of 1.4-2.2 nm over a $50 \times 50 \mu \mathrm{m}^{2}$ area. The RMS surface roughness is characterized by the following equation:

$$
X_{r m s}=\sqrt{\frac{\sum\left(X_{t}-X_{a v e}\right)^{2}}{N}},
$$

where $X_{i}, X_{\text {ave }}$, and $N$ are measured values, average of the measured values, and total number of measurement for a specific area, respectively. The film thicknesses of different layers are given in Fig. 1(a).

\section{Organic materials properties and experiments}

The light-emissive materials used in this work are based on a family of fluorene-containing alternating conjugated co-polymers developed by Dow Chemical Corp. The chemical structures of the materials are described in Ref. 22. Table 1 shows a summary of the materials properties used in this work. Figure 2(a) shows the absorption spectra of the red-, green-, and blue-light-emitting polymers. The absorption of 


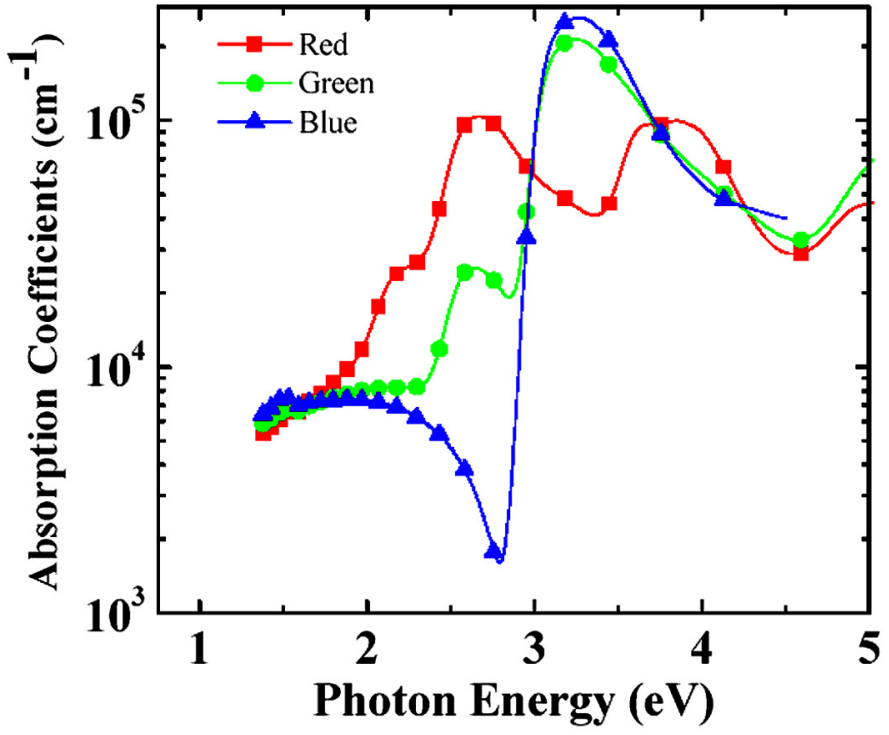

(a)

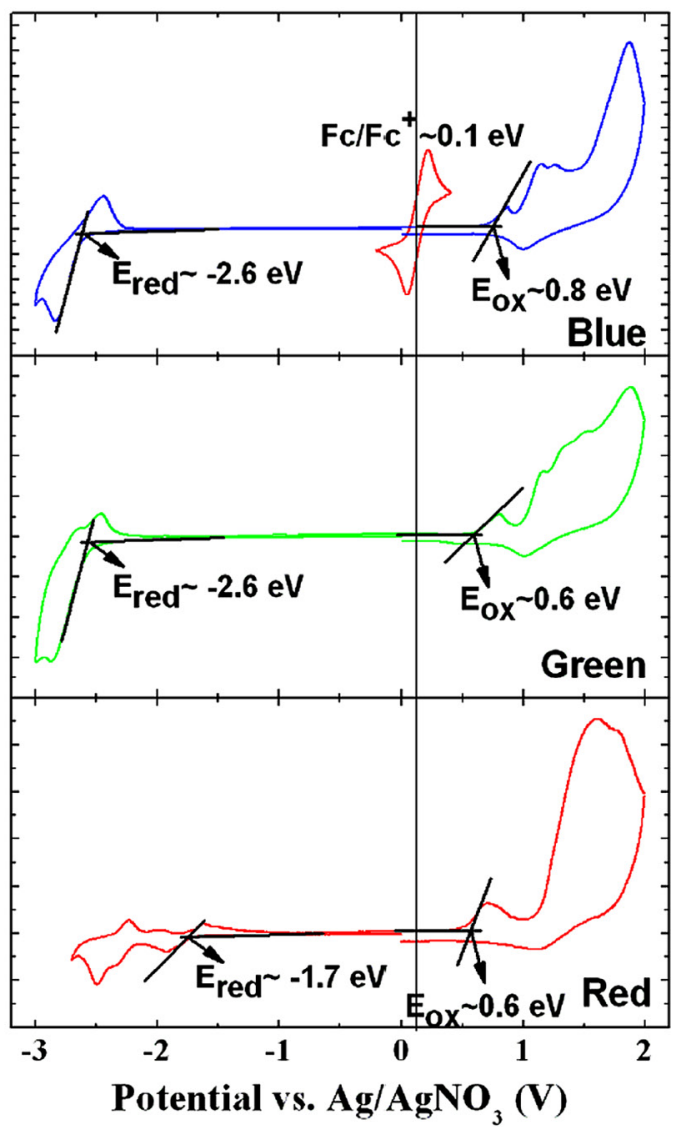

(b)

FIGURE 2 - (a) Optical absorption spectra and (b) cyclic voltammograms of the red-, green-, and blue-light-emitting polymer thin films.

light by a molecule comes from the electronic transitions between different electronic energy levels of a molecule [i.e., highest occupied molecular orbital (HOMO) and lowest unoccupied molecular orbital (LUMO) levels]. To derive the information of HOMO and LUMO, we used cyclic voltammetry $(\mathrm{CV})$ measurement methods described below [Fig. 2(b)]. We then used this information to construct the energy-band diagram of the fabricated devices (Fig. 3). The photoluminescence quantum efficiency (PLQE) of the polymers is obtained by the integrating sphere method. ${ }^{23}$ The integrated photoluminescence (PL) and electroluminescence (EL) spectra are collected by a JY spectroradiometer system equipped with an integrating sphere as the input optics. ${ }^{24}$ An integrated system consisting of an INS250 integrating sphere coated with barium sulfate from the International Light (measure L), a programmable Keithley 617 electrometer (measure I), and a $230-\mathrm{V}$ source (source V) were used for simultaneously collecting the optical and electrical data controlled by a homemade program written in the Labview language. ${ }^{24}$ The integrating sphere was calibrated to measure the total photon flux entering the sphere system. Therefore, the current-voltage-luminous flux $(I-V-L)$ relationship was obtained. We then derived the luminance from the measured data by assuming a Lambertian angular distribution of the emission, which was confirmed experimentally. ${ }^{34}$
TABLE 1 - Summary of material and device properties.

\begin{tabular}{|c|c|c|c|}
\hline & Red & Green & Blue \\
\hline $\mathrm{HOMO}(\mathrm{eV})$ & 5.3 & 5.3 & 5.5 \\
\hline LUMO (eV) & 3.0 & 2.1 & 2.1 \\
\hline PLQE & $54 \pm 5 \%$ & $74 \pm 5 \%$ & $31 \pm 5 \%$ \\
\hline PL peak (nm) & 668 & 547 & 467 \\
\hline EL peak $(\mathrm{nm})$ & 661 & 549 & 469 \\
\hline Active layers $^{a}$ & $\mathrm{HIL} / \mathrm{BFE} / \operatorname{Red}^{b}$ & HIL/Green & HIL/Blue \\
\hline $\mathrm{PE}_{\max }(\operatorname{lm} / \mathrm{W})^{c}$ & 0.6 & 9.9 & 0.5 \\
\hline $\mathrm{EE}_{\max }(\mathrm{cd} / \mathrm{A})^{c}$ & 0.7 & 10 & 1.7 \\
\hline $\mathrm{EQE}_{\max }(\%)^{c}$ & 1.5 & 2.9 & 1.2 \\
\hline $\mathrm{EL} \mathrm{CIE}^{d}$ & $(0.67,0.32)$ & $(0.42,0.56)$ & $(0.17,0.22)$ \\
\hline
\end{tabular}

${ }^{a}$ Device structures used for this study are ITO/active layers $/ \mathrm{Ca} / \mathrm{Al}$.

${ }^{b}$ HIL: hole injection layer (PEDOT:PSS), BFE: poly(9, 9'-dioctyl fluorene-2,7-diyl)-copoly(diphenyl-p-tolyl-amine-4,4'-diyl.

${ }^{c} \mathrm{PE}_{\max }, \mathrm{EE}_{\max }$, and $\mathrm{EQE}_{\max }$ are maximum power efficiency, emission efficiency, and external quantum efficiency, respectively.

${ }^{d} \mathrm{EL} \mathrm{CIE}$ is the CIE coordinate of the electroluminescence spectra.

From Table 1, we can conclude that the EL spectra of the red-, green-, and blue-light-emitting materials show a similar peak position with slight shift in position $(<7 \mathrm{~nm})$ in comparison with their PL spectra. 


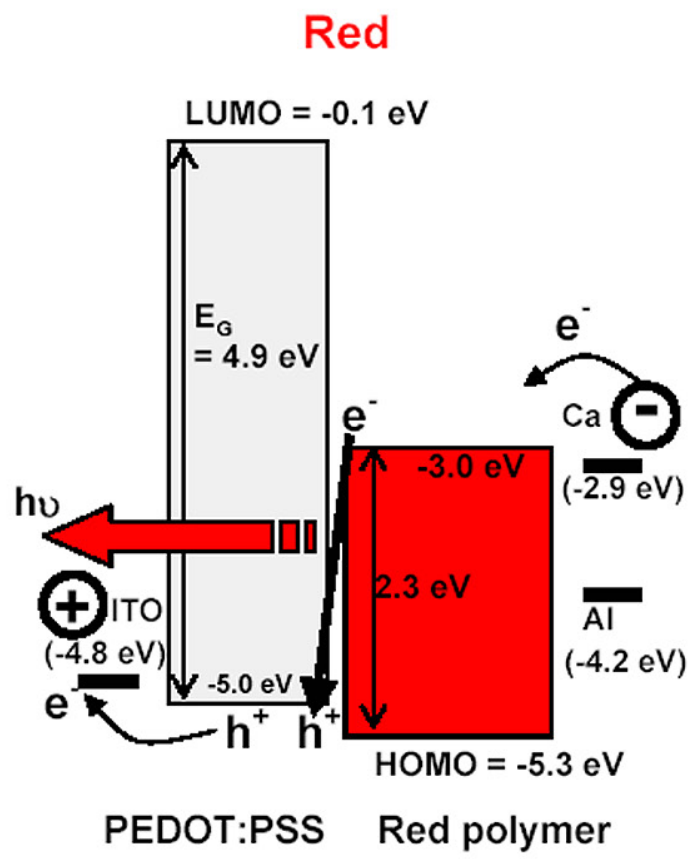

Green

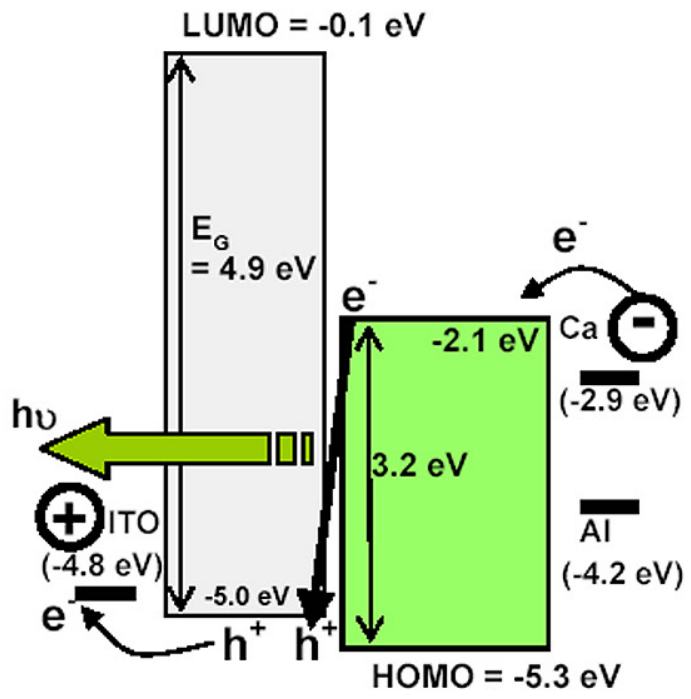

\section{PEDOT:PSS Green polymer}

\section{Blue}

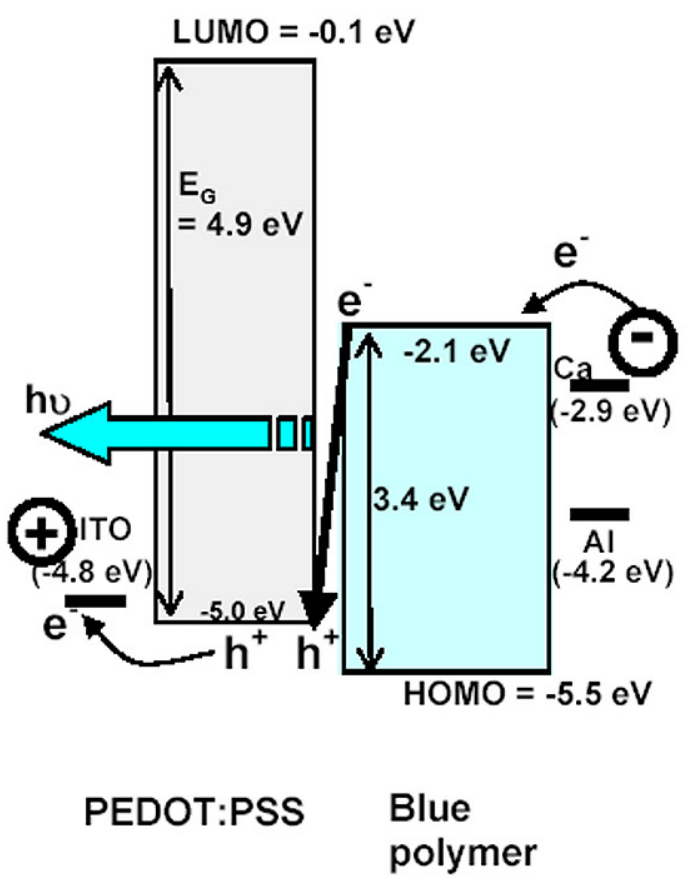

FIGURE 3 - The PLED energy-band diagram for green and blue PLEDs.

\subsection{Electrochemical properties}

The oxidation and reduction potentials of materials were obtained by cyclic voltammetry measurements. The cyclic voltammetry (CV) data were measured with a CHI 660A model from CH Instruments. The light-emitting polymer films were deposited on a platinum wire electrode in an electrolyte solution of $\mathrm{TBAPF}_{6}(0.1 \mathrm{M})$ in $\mathrm{CH}_{3} \mathrm{CN}$. The potentials were measured relative to an $\mathrm{Ag} / \mathrm{Ag}^{+}$reference electrode (scan rate of $0.5 \mathrm{~V} / \mathrm{sec}$ ).

The ionization potential $\left(I_{p}\right)$ and electron affinity $\left(E_{\mathrm{a}}\right)$ were derived from the respective edges of the oxidation and reduction potentials obtained by cyclic voltammetry measurements. In Fig. 2(b), we observed oxidation and reduction 

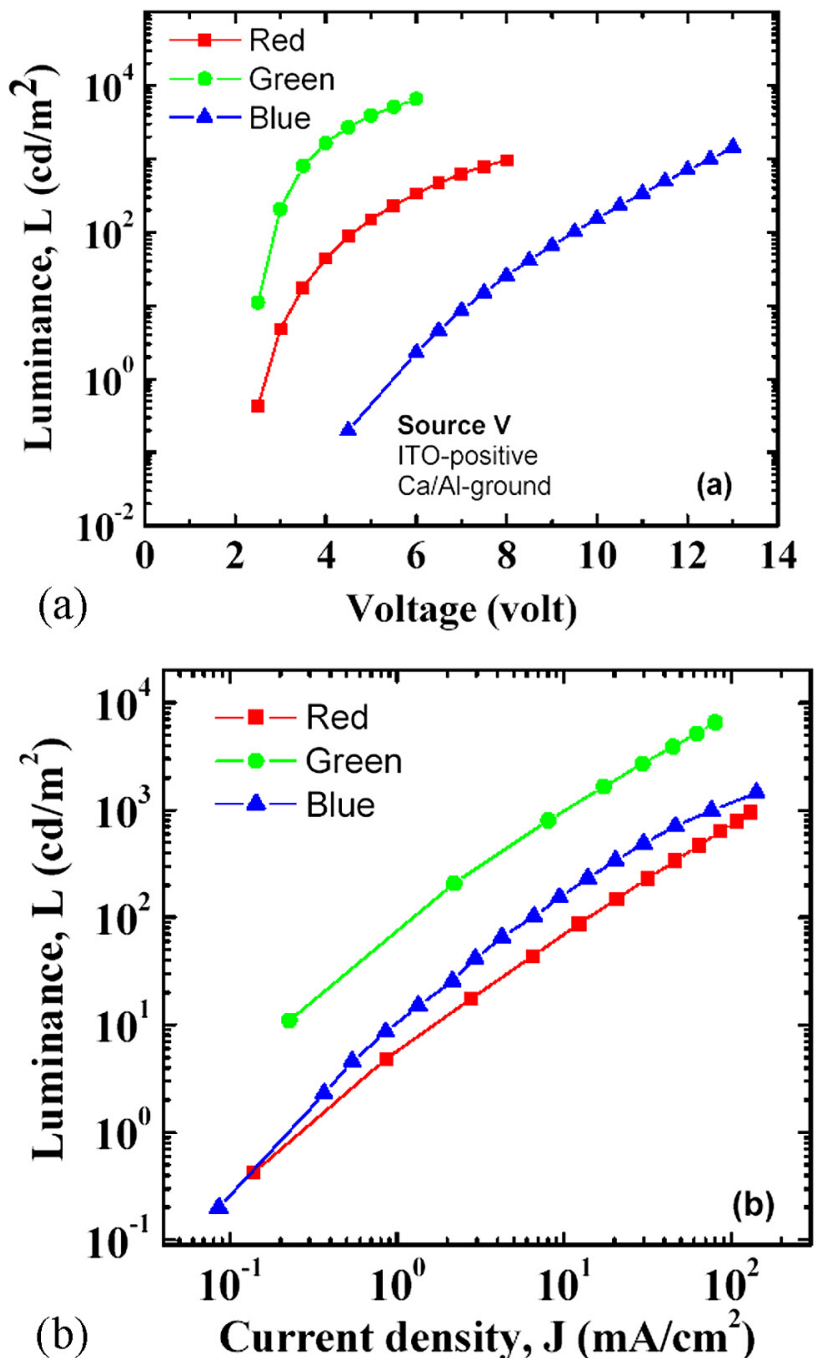

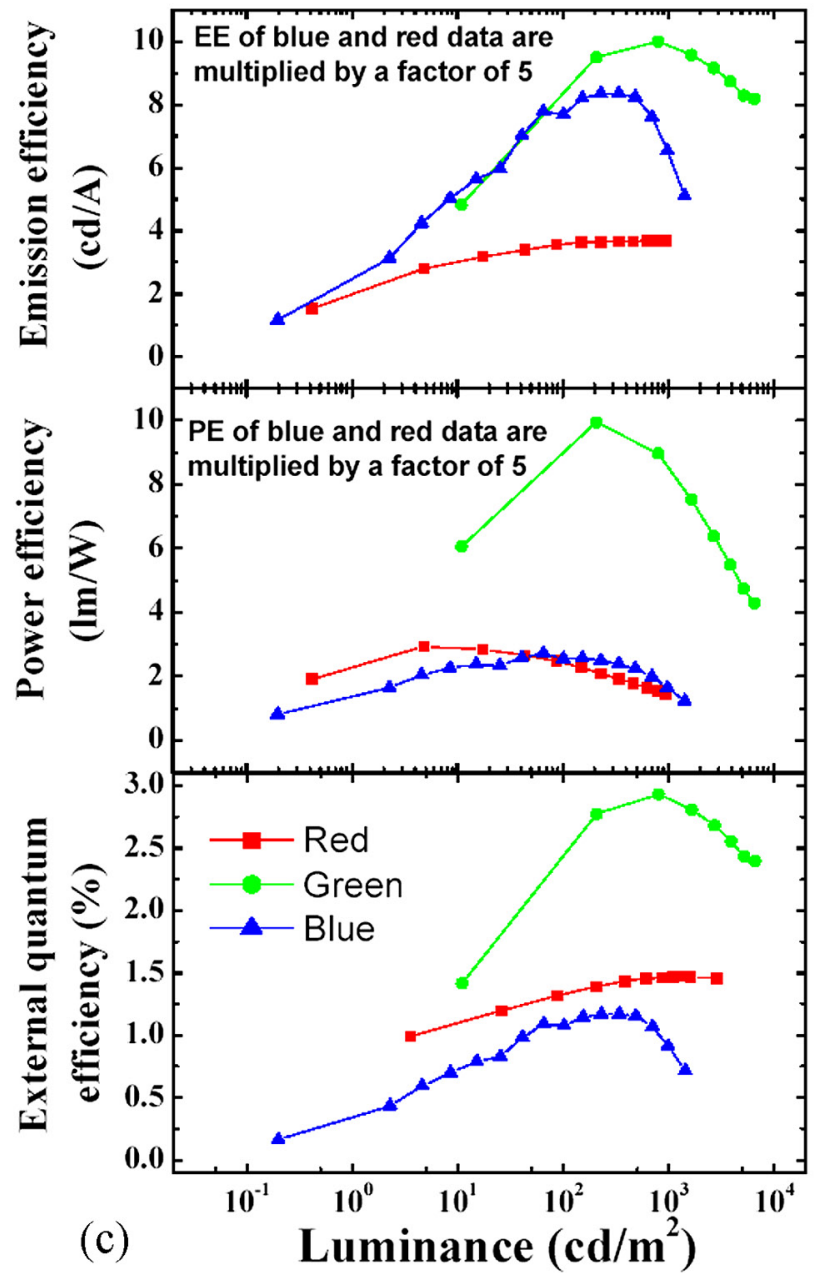

FIGURE 4 - (a) Luminance $(L)$-voltage ( $V$ ), (b) luminance $(L)$-current density $(f)$, and (c) emission efficiency, power efficiency, and external quantum efficiency vs. luminance of the red-, green-, and blue-light-emitting devices on a plastic substrate.

peaks for the polymer thin films. Because it is believed that the edge of the oxidation potential $\left(E_{O x}\right)$ represents the triggering point of the oxidation process, ${ }^{17}$ we used this value to define the HOMO level of our emissive polymer. The edge of the reduction potential $\left(E_{\text {red }}\right)$ was used to calculate the LUMO level of the polymer. It is usually assumed that the Ferrocene/Ferrocenium $\left(\mathrm{Fc} / \mathrm{Fc}^{+}\right)$oxidation potential $\left(E_{1 / 2}\right)$ corresponds to $4.8 \mathrm{eV}$ in reference to the vacuum. ${ }^{17}$ Hence, this factor was used to estimate the $I_{p}$ and $E_{a}$ of the polymeric materials, thus defining the HOMO and LUMO levels, respectively.

$$
\begin{gathered}
I_{p}=-\left(E_{o x}-E_{1 / 2}\right)-4.8, \\
E_{a}=-\left(E_{\text {red }}-E_{1 / 2}\right)-4.8 .
\end{gathered}
$$

Different values for the light-emissive polymers are listed in Table 1. In Fig. 2(b), the edge of the oxidation potential of blue polymer is $E_{o x} \sim 0.8 \mathrm{~V} v s . \mathrm{Ag} / \mathrm{AgNO}_{3} \mathrm{cou}-$ ple; the edge of the reduction potential of blue polymer is $E_{\text {red }} \sim-2.6 \mathrm{~V} v s . \mathrm{Ag} / \mathrm{AgNO}_{3}$; and the half-oxidation potential of $\mathrm{Fc} / \mathrm{Fc}^{+}$is $E_{1 / 2} \sim 0.1 \mathrm{~V}$ vs. $\mathrm{Ag} / \mathrm{AgNO}_{3}$. Therefore, $I_{p}$ and $E_{a}$ are -5.5 and $-2.1 \mathrm{eV}$, respectively. By a similar procedure, we can also obtain $I_{p}$ and $E_{a}$ for red- and green-light-emitting materials.

\section{Device optoelectronic properties}

To achieve PLEDs with high efficiency and long lifetime, one basic requirement is needed: balanced electron and hole current, which results from balanced charge injection and transport. ${ }^{25}$ The simplest PLED structure consists of a light-emitting layer (LEL) sandwiched between an anode and a cathode. Since the anode/LEL and cathode/LEL junctions have different barrier heights (Fig. 3), it is expected that the density of generated electrons and holes (produced by electrons extraction mechanism at the anode) will be different. In addition, the carriers have different mobilities in the organic polymers. ${ }^{26,27}$ Hence, when the two types of carriers are generated in the LEL, they do not recombine in identical proportions and the recombination processes can 
take place near the electrode that produces the least mobile carriers. This will lead to a poor efficiency in luminescence because a high number of majority carriers reach the opposite electrode without encountering the minority carriers. In addition, a large density of metal- induced defects near the cathode interface can lead to non-radiative recombination. ${ }^{22}$

The multilayer device structure effectively enhances device light-emission efficiency by incorporating an electron transport/injection layer (ETL/EIL) between an active emissive layer (EL) and a cathode ${ }^{28}$ and/or a hole transport/injection layer (HTL/HIL) between an EL and an anode. ${ }^{29}$ The ETL/EIL and HTL/HIL could reduce the effective energy barriers for electrons and holes, respectively. Therefore, the charge creation efficiency and subsequently their recombination rate are improved. In addition, the recombination region is moving away from the anode and cathode surfaces where a large density of defects might present, thus reducing the non-radiative recombination at the electrode surface. Our laboratory has employed the multi-layer device structure to optimize the device efficiency with a careful selection of materials and solvents to avoid damage of the polymer layers during the layer-by-layer wet spin-coating process. Table 1 shows the device results obtained in our laboratory based on red-, green-, and bluelight-emitting polymers.

For all studied red, green, and blue polymer devices, the ITO and calcium $(\mathrm{Ca}) /$ aluminum $(\mathrm{Al})$ were used as anode and cathode electrodes, respectively. The poly(3,4-ethylenedioxythiophene) (PEDOT) doped with the poly(styrenesulfonate) (PSS) was used as the hole-injection layer (HIL). When a forward bias (ITO - positive and $\mathrm{Ca}$ - ground) is applied, the established electric field between ITO and Ca (Fig. 3) triggers (a) electron injection from Ca cathode into polymer LUMO levels and (b) electron extraction from PEDOT:PSS HOMO levels into ITO anode. The electron extraction process leaves a positive charge (hole) in PEDOT: PSS HOMO levels, which can be referred to as "hole injection" from ITO electrode into PEDOT:PSS HOMO levels. When the electric field is high enough, the electrons and holes will drift/diffuse toward each other from polymer chain to polymer chain or/and within a polymer chain. Finally, a number of the electrons and holes will recombine with each other and will decay radiatively to produce visible light emission.

For red PLEDs, an organic hole-transport layer (HTL) is inserted between PEDOT:PSS hole-injection layer (HIL) and light-emitting layer (LEL). Since the highest occupied molecular orbital of the HTL (HOMO 5.3 $\mathrm{eV}$ ) is located between those of HIL and LEL, the insertion of HTL reduces the effective HOMO level offset between HIL and LEL, reducing the device operation voltage and producing comparable or better device efficiencies in comparison with the conventional PEDOT:PSS-only devices.

Device efficiency and chromaticity are the most important opto-electronic properties of PLEDs. Typical device efficiencies obtained for red, green, and blue PLEDs in our laboratory are shown in Fig. 4. The device efficiencies could be expressed in three common used units: (1) emission efficiency (EE), (2) power efficiency (PE), and (3) external quantum efficiency (EQE). EE is defined as the ratio of the output light intensity (cd) to the input current intensity (A); $\mathrm{PE}$ is defined as the ratio of the total output of optical power $(\mathrm{lm})$ to the input electrical power $(\mathrm{W})$; and EQE is defined as the ratio of the total output of photons to the input electrons in the following equations:

$$
\begin{gathered}
\mathrm{EE}=\frac{\text { output luminous intensity through the PLED front }(\mathrm{cd})}{\text { input current flowing through the PLED }(\mathrm{A})}, \\
\mathrm{PE}=\frac{\text { output luminous flux through the PLED front }(\mathrm{lm})}{\text { input electrical power through the PLED }(\mathrm{W})}, \\
\mathrm{EQE}=\frac{\text { output \# of photons through the PLED front }}{\text { input \# of electrons through the PLED }} .
\end{gathered}
$$

For the red polymer device, the staircase-like increase of the HOMO level - reduced effective hole injection (or electron extraction) energy barrier - between anode and LEL enhances the device efficiencies. For all the PLEDs, a low-work-function calcium cathode, which reduces the effective electron-injection barrier and enhances the device efficiencies, was used. The turn-on voltage, defined at $1 \mathrm{~cd} / \mathrm{m}^{2}$, is $\sim 2.3, \sim 2.1$, and $\sim 5.4 \mathrm{~V}$ for red-, green-, and blue-lightemitting devices, respectively.

It is difficult to directly compare our device efficiency with other group's data because the device efficiency is highly sensitive to the device processing ${ }^{30,31}$ and measurement conditions. ${ }^{24}$ As a reference, Cambridge Display Technology (CDT) has reported that the device emission efficiencies $\left(\right.$ at $100 \mathrm{~cd} / \mathrm{m}^{2}$ ) on a glass substrate are $1.7,9$, and $2.0 \mathrm{~cd} / \mathrm{A}$ for red, green, and blue PLEDs, respectively. ${ }^{32} \mathrm{As}$ can be seen in Fig. 4(c), our device emission efficiencies (at $100 \mathrm{~cd} / \mathrm{m}^{2}$ ) on a plastic substrate are $0.7,8.3$, and $1.5 \mathrm{~cd} / \mathrm{A}$ for red, green, and blue PLEDs, respectively.

Figure 5(a) shows the electroluminescence (EL) spectra for the red, green, and blue PLEDs. We further calculated their corresponding CIE (Commission Internationale de I'Éclairage) color coordinates based on CIE 1931 chromaticity calculations. ${ }^{33} \mathrm{We}$ achieved the CIE coordinates of the PLEDs in a wide range of the visible spectra when employing different emissive polymers [Fig. 5(b)]. CIE coordinates of our PLEDs are red $(0.67,0.32)$, green $(0.42,0.56)$, and blue $(0.17,0.22)$. As a reference, the CIE coordinates from National Television System Committee (NTSC) are red $(0.67,0.33)$, green $(0.21,0.71)$, and blue $(0.14,0.08)$. Therefore, the color-mapping area of our colors is $\sim 74 \%$ of that of NTSC's.

\section{Angular distribution of light emission}

We measured the electroluminescence spectra of the green PLEDs at different angles. ${ }^{34}$ From this we concluded that 

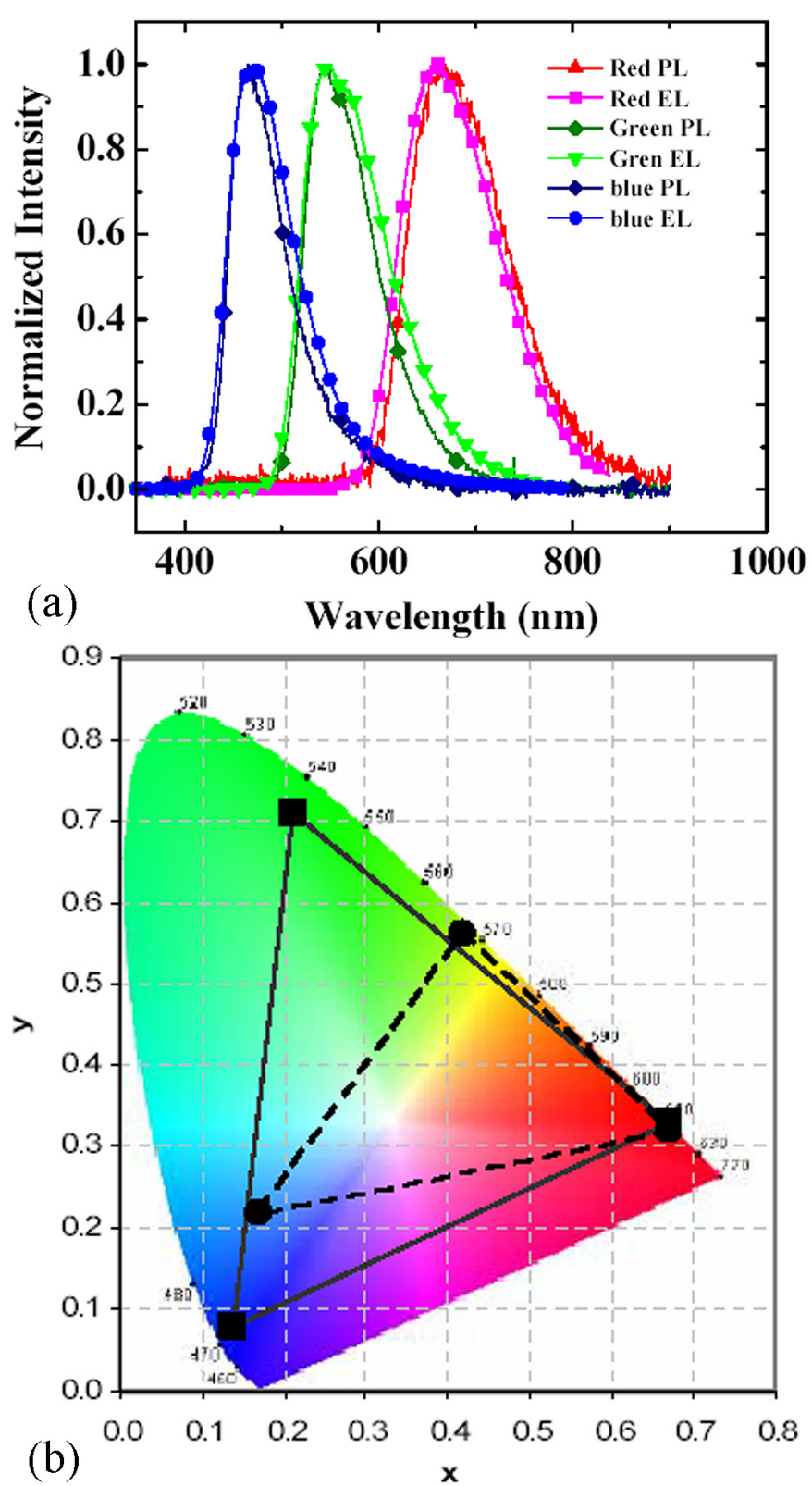

FIGURE 5 - (a) Photoluminescence spectra of materials and electroluminescence spectra of the red, green, and blue PLEDs on a plastic substrate. (b) The CIE color coordinates of the red, green, and blue based on NTSC (solid line) and our PLEDs (dash line).

the shape of the measured electroluminescence spectra does not change with the measured angle. We then integrated the spectral radiant intensity over the entire spectra region at each angle and we normalized integrated radiant intensity to its value at the normal angle $\left(\theta=0^{\circ}\right)$ to the plane of the PLED. The variation of the normalized photon density for different angles is shown as curve (b) in Fig. 6. The experimental light-emission angular distribution of our green PLED is very close to that of a Lambertian light source, in agreement with published results. ${ }^{35}$ Also we have obtained the best agreement between experimental and Monte Carlo simulated results ${ }^{34}$ when we take into account refractions in the PLED, back reflection from the cathode,

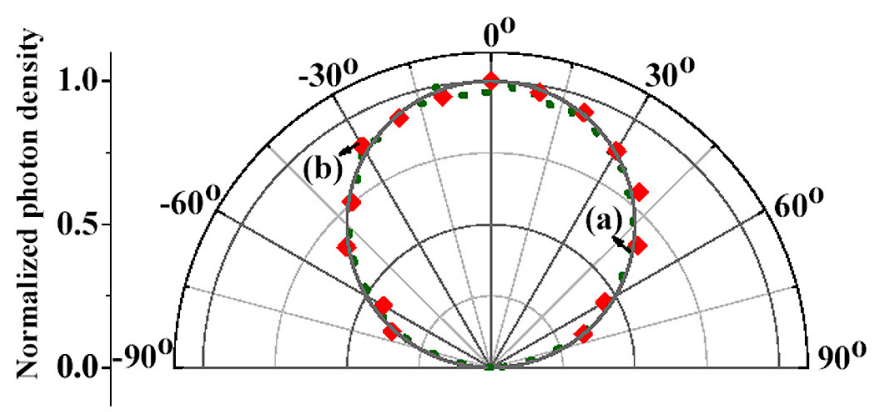

FIGURE 6 - (a) Simulated and (b) experimental angular distribution of the green PLEDs on a plastic substrate. The background gray solid line represents the Lambertian angular distribution of the light source.

absorption in polymer layers, and interference effect in the ITO thin films. Based on these results, we concluded that all effects must be taken into consideration when we compare simulated and experimental PLED opto-electronic characteristics. For PLED to be used in flat-panel-display application, it is very desirable to have the Lambertian viewing-angle dependence of the light emission because it means that the display luminance is constant irregardless of the viewing angle.

\section{SPICE modeling of PLEDs}

In general, two approaches have been proposed to calculate the current density $(J)$-voltage $(V)$ characteristics of the PLEDs. The first approach utilized physical models to describe the carrier-conduction mechanisms which can be responsible for the $J-V$ curves of PLEDs. These models include a carrier-injection-dominated mechanism such as FowlerNordheim $(F-N)$ tunneling ${ }^{36}$ or a carrier-transport-dominated mechanism such as space-charge-limited current $(\mathrm{SCLC})^{37}$ or trapped charge-limited current (TLC). ${ }^{38}$ The $J-V$ characteristics of these models are described by the following equations:

$$
\begin{aligned}
& \ln \left(\frac{J}{F^{2}}\right) \propto-\frac{1}{F}, \quad F=\frac{V}{d}(\mathrm{~F}-\mathrm{N} \text { tunneling }), \\
& J \propto V^{2}(\mathrm{SCLC}), \\
& J \propto V^{m+1}, m>1 \text { (TLC) }
\end{aligned}
$$

where $d$ is thickness of PLEDs and $F$ is the applied electric filed. Since the $J-V$ characteristics of our PLEDs are limited by both carrier generation at the polymer-electrode interface and conduction in polymer thin films, ${ }^{39}$ the $J-V$ characteristics of PLEDs can not be simply fitted to one of the above relationships when the current density of PLEDs ranges over several orders of magnitude $\left(10^{-5}-10^{2} \mathrm{~mA} / \mathrm{cm}^{2}\right)$. An allcarrier-transport mechanism should be considered in practical devices, which is a rather complex problem.

The second approach utilized engineering circuit models to simulate the $J-V$ characteristics of PLEDs. This approach is used when PLED modeling is necessary for the design of a flat-panel display. Bender et al. proposed an 


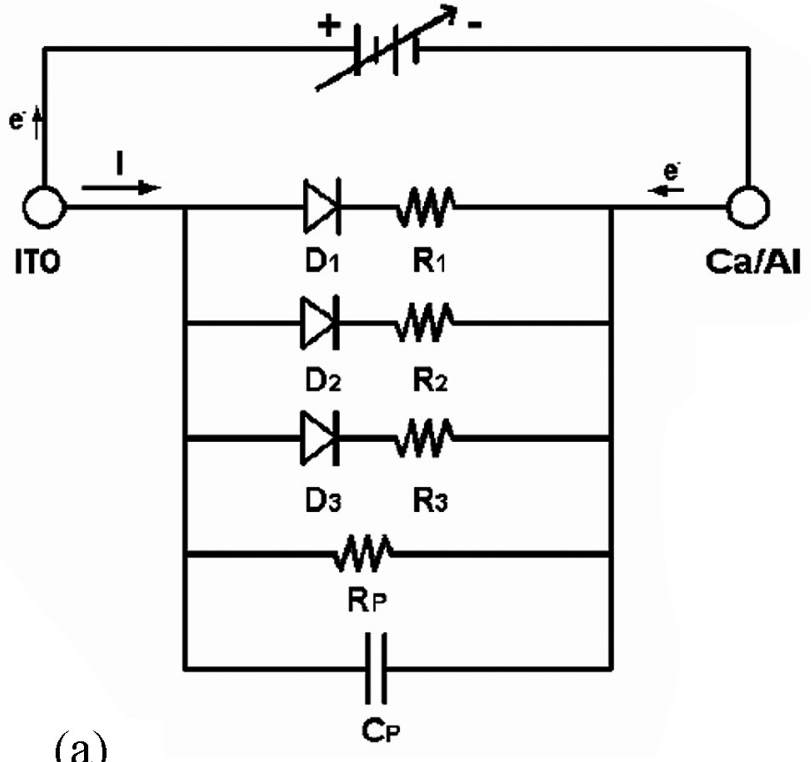

(a)

FIGURE 7 - (a) Proposed equivalent circuit model for PLEDs. (b) Experimental and simulated $J-V$ characteristics of red, green, and blue PLEDs.

equivalent-circuit configuration to describe the injection and bulk-limited current in OLEDs. ${ }^{40}$ Bonnassieux et al. reported an OLED SPICE (Simulation Program with Integrated Circuit Emphasis) model in a passive-matrix configuration taking into consideration the electrical coupling of the pixels. ${ }^{41}$ We have also used an engineering circuit model approach to simulate the PLEDs current density-voltage $(J-V)$ characteristics to be used in active-matrix organic light-emitting displays.

Since the dark $J-V$ characteristics of our PLEDs are limited by both carrier injection at the contacts and bulk conduction, ${ }^{39}$ in this experiment, the $J-V$ characteristics could not be simply described by a single diode behavior. Three parallel-connected diodes $\left(D_{1}, D_{2}\right.$, and $\left.D_{3}\right)$ with serial resistors $\left(R_{1}, R_{2}\right.$, and $\left.R_{3}\right)$, parallel resistor $R_{\mathrm{P}}$, and capacitor $C_{\mathrm{P}}$ need to be considered in this work to accurately fit the PLED experimental $J-V$ curves [Fig. 7(a)].

We used the following diode current equation for each diode:

$$
J=J_{S}\left(e^{q V_{a} / N k_{B} T}-1\right),
$$

where $J_{s}$ is the reverse saturation current of the diode, $q$ is the elemental charge, $V_{a}$ is the applied bias, $N$ is an ideality factor, $k_{\mathrm{B}}$ is the Boltzmann constant, and $T$ is temperature. For our calculation [Fig. 7(b)], the fitting parameters for a

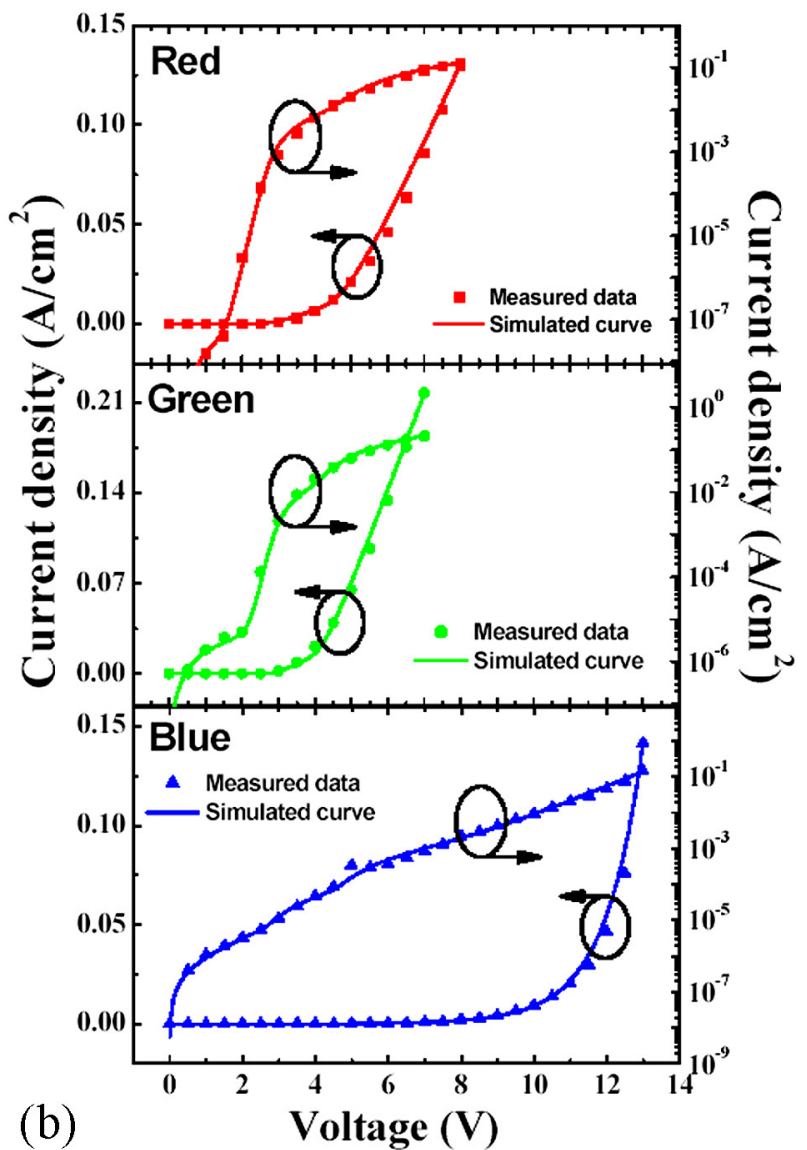

green PLED are: $D_{1}\left(J_{S}=3.0 e-8 \mathrm{~A}, N=3.1\right), D_{2}\left(J_{S}=\right.$ $3.0 e-14 \mathrm{~A}, N=4.5), D_{3}\left(J_{S}=1.0 e-20 \mathrm{~A}, N=3.9\right), R_{1}=16.4$ $\Omega, R_{2}=60 \Omega, R_{3}=400 \mathrm{~K} \Omega$, and $R_{P}=10 \mathrm{M} \Omega$; for a red PLED: $D_{1}\left(J_{S}=1.0 e-12 \mathrm{~A}, N=8\right), D_{2}\left(J_{S}=9.0 e-14 \mathrm{~A}, N=\right.$ 4.6), $D_{3}\left(J_{S}=9.0 e-29 \mathrm{~A}, N=0.5\right), R_{1}=2.8 \Omega, R_{2}=150 \Omega$, $R_{3}=30 \mathrm{M} \Omega$, and $R_{P}=500 \mathrm{M} \Omega$; and for a blue PLED: $D_{1}$ $\left(J_{S}=4.9 e-7 \mathrm{~A}, N=40\right), D_{2}\left(J_{S}=5.0 e-25 \mathrm{~A}, N=4\right), D_{3}\left(J_{S}=\right.$ $3.5 e-18 \mathrm{~A}, N=3.9), R_{1}=0.01 \Omega, R_{2}=4 \mathrm{k} \Omega, R_{3}=50 \mathrm{k} \Omega$, and $R_{P}=10 \mathrm{M} \Omega$. To be able to perform the simulation of transient response of PLEDs, a parallel capacitor is also added. The capacitance value of a PLED is estimated from the capacitance value of the light-emissive layer (i.e. $C_{P}=\varepsilon_{r} \varepsilon_{o} \mathrm{~A} / d$, where $\varepsilon_{r}$ is the relative dielectric constant, $d$ is the thickness $(\sim 1000 \AA), A$ is the active device area $\left(6 \mathrm{~mm}^{2}\right)$, and $\varepsilon_{o}$ is the dielectric constant of free space $=8.854 e-12 \mathrm{~F} / \mathrm{m}^{2}$ ). The relative dielectric constants of red, green, and blue polymers are measured to be 2.2, 2.3, and 2.1; therefore, the capacitance values of red, green, and blue PLEDs are 1.22, 1.17 , and $1.12 \mathrm{nF}$, respectively. It is noted that the capacitance value of a green PLED is measured to be $1.1 \mathrm{nF}$ at a frequency of $10 \mathrm{kHz}$ for a PLED size of $6 \mathrm{~mm}^{2}$, which is consistent with the estimated values. For all PLEDs operated in the low-voltage regime (before the turn-on region), the current mainly flows through the parallel connected diode $D_{1}$. After the PLED is turned on and emits light, $D_{2}$ dominates the $J-V$ characteristics. When the PLED is oper- 
ated in a high-voltage regime, $D_{3}$ dominates the $J-V$ characteristic while the current contributions flowing through $D_{1}$ and $D_{2}$ are negligible.

The difference between simulated and measured data is negligible before PLEDs are turned on as shown in logscale in Fig. 7(b). The maximum differences after PLEDs are turned on and emit light are less than $16 \%$ for all RGB colors. It should be noted that the fitting parameters in the diode-current equation, which is introduced above, have to be carefully chosen to achieve the best possible match between calculated and experimental results. In addition, the reverse saturation current $J_{S}$ has the most significant effect when fitting the $J-V$ curves in the low-voltage regime, and the serial resistors have important effect at the high-voltage regime.

In the band diagram shown in Fig. 3, the blue PLED has a very similar band structure as the green PLED. However, the $J-V$ characteristic curve for blue PLED shown in Fig. 7(b) is very different from green PLED. The turn-on voltage of blue PLED is about $10 \mathrm{~V}$, which is $7 \mathrm{~V}$ higher than the green PLED, and we mainly adjust the parameters of $D 1$ to fit the curve of blue PLED. This result suggests that the $J-V$ model for PLEDs not only depends on the injection-dominated mechanism, but also on the carrier-transport mechanism through bulk. The three-parallel-diode model described here is a best approach to model $J-V$ characteristics of the PLEDs to be used in the design of AMPLEDs.

\section{Conclusions}

We reported on the opto-electronic properties of red, green, and blue poly(fluorene) co-polymer light-emitting devices (PLEDs) fabricated on a flexible plastic substrate having a water vapor and oxygen transmission rate of less than $10^{-5} \mathrm{~g} / \mathrm{cm}^{2}$-day-atm and $10^{-7} \mathrm{cc} / \mathrm{cm}^{2}$-day-atm, respectively. We obtained a wide color gamut and a maximum emission efficiency of $0.7,10$, and $1.7 \mathrm{~cd} / \mathrm{A}$ for red, green, and blue PLEDs, respectively. Finally, a simple SPICE equivalent circuit model was used to simulate the PLED current-density-voltage characteristics.

\section{Acknowledgment}

The authors would like to thank Mr. Aaron Johnson for the discussion on cyclic voltammetry measurements and $\mathrm{Mr}$. Hojin Lee for discussion on the photoluminescence measurement. This work was supported by NIH and DARPA grants. The organic polymers used in this work were provided by Dow Chemical Corp. (Midland, MI).

\section{References}

1 Y Hong, J-Y Nahm, and J Kanicki, “100 dpi 4-a-Si:H TFTs active-matrix organic polymer light-emitting display," IEEE J Select Topics Quantum Electron 10, 16-25 (2004).
2 T Tsujimura, Y Kobayashi, K Murayama, A Tanaka, M Morooka, E Fukumoto, H Fujimoto, J Sekine, K Kanoh, K Takeda, K Miwa, M Asano, N Ikeda, S Kohara, S Ono, C-T Chung, R-M Chen, J-W Chung, C-W Huang, H-R Guo, C-C Yang, C-C Hsu, H-J Huang, W Riess, H Riel, S Karg, T Beierlein, D Gundlach, S Alvarado, C Rost, P Mueller, F Libsch, M Mastro, R Polastre, A Lien, J Sanford, and R Kaufman, "A 20-inch OLED display driven by super-amorphous-silicon technology,” SID Symposium Digest Tech Papers 34, 6-9 (2003).

3 J-J Lih, C-F Sung, M S Weaver, M Hack, and J J Brown, "A Phosphorescent active-matrix OLED display driven by amorphous silicon backplane," SID Symposium Digest Tech Papers 34, 14-17 (2003).

4 T Sasaoka, M Sekiya, A Yumoto, J Yamada, T Hirano, Y Iwase, T Yamada, T Ishibashi, T Mori, M Asano, S Tamura, and T Urabe, “A 13.0-inch AM-OLED display with top emitting structure and adaptive current mode programmed pixel circuit," SID Symposium Digest Tech Papers 32, 384-387 (2001).

5 O Prache, "Active matrix molecular OLED microdisplays," Displays 22, 49-56 (2001).

6 J J Brown and G Yu, "Flexible OLED displays," SID Symposium Digest Tech Papers 34, 855-872 (2003).

7 A Sugimoto, H Ochi, S Fujimura, A Yoshida, T Miyadera, and M Tsuchida, "Flexible OLED displays using plastic substrates," IEEE J Select Topics Quantum Electron 10, 107-114 (2004).

8 A B Chwang, M A Rothman, S Y Mao, R H Hewitt, M S Weaver, J A Silvernail, K Rajan, M Hack, J J Brown, X Chu, L Moro, T Krajewski, and N Rutherford, "Thin film encapsulated flexible OLED displays," Appl Phys Lett 83, 413-415 (2003).

9 S Utsunomiya, T Kamakura, M Kasuga, M Kimura, W Miyazawa, S Inoue, and T Shimoda, "3-inch full-color OLED display using a plastic substrate,” SID Symposium Digest Tech Papers 34, 864-867 (2003).

10 J S Lewis and M S Weaver, "Thin-film permeation-barrier technology for flexible organic light-emitting devices," IEEE J Select Topics Quantum Electron 10, 45-57 (2004).

11 G Gustafsson, Y Cao, G M Treacy, F Kavetter, N Colaneri, and A J Heeger, "Flexible light-emitting diodes made from soluble conducting polymers," Nature 357, 477-479 (1992).

12 G Gu, P E Burrows, S Venkatesh, S R Forrest, and M E Thompson, "Vacuum-deposited, nonpolymeric flexible organic light-emitting devices," Opt Lett 22, 172-174 (1997).

13 H Lim, W J Cho, C S Ha, S Ando, Y K Kim, C H Park, and K Lee, "Flexible organic electroluminescent devices based on fluorine-containing colorless polyimide substrates," Adv Mater 14, 1275-1279 (2002)

14 Y Hong, Z He, N S Lennhoff, D A Banach, and J Kanicki, “Transparent flexible plastic substrates for organic light-emitting devices," J Electron Mater 33, 312-320 (2004).

15 P E Burrows, G L Graff, M E Gross, P M Martin, M K Shi, M Hall, E Mast, C Bonham, W Bennett, and M B Sullivan, "Ultra barrier flexible substrates for flat panel displays,” Displays 22, 65-69 (2001).

16 Y Hong, Z Hong and J Kanicki, "Materials and devices structures for high performance poly OLEDs on flexible plastic substrates," Proc SPIE 4105, 356-361 (2000).

17 Y Hong, Z He, S-J Lee, and J Kanicki, “Air-stable organic polymer red light-emitting devices on flexible plastic substrates," Proc SPIE 4464, 329-335 (2001).

18 Y He and J Kanicki, "High efficiency organic polymer light-emitting devices on the flexible plastic substrates," Appl Phys Lett 76, No. 6, 661-663 (2000).

19 H C Choi, Y Z Chu, L S Heath, and W K Smyth, U.S. Patent \#6,379,509 (30 April 2002).

20 N S Lennhoff and J Ram, U.S. Patent Application \#20020182386.

21 Y Aoshima, M Miyazaki, K Sato, Y kao, S Takaki, and K Adachi, "Improvement of alkali durability of silver-based multilayer coatings for use in flat panel displays," Jpn J Appl Phys 40, 4166-4170 (2001).

22 M T Bernius, M Inbasekaran, J O'Brien, and W Wu, "Progress with light-emitting polymers," Adv Mater 12, 1737-1750 (2000).

$23 \mathrm{~J}$ C de Mello, H F Wittmann, and R H Friend, "An improved experimental determination of external photoluminescence quantum efficiency," Adv Mater 9, 230-232 (1997).

24 Y Hong and J Kanicki, "Integrating sphere CCD-based measurement method for organic light-emitting devices," Rev Sci Instru 74, 3572-3575 (2003).

25 N K Patel, S Cinà, and J H Burroughes, "High-efficiency organic light-emitting diodes," IEEE J Select Topics Quantum Electron 8, 346-361 (2002). 
26 M Redecker, D D C Bradley, M Inbasekaran, and E P Woo, "Nondispersive hole transport in an electroluminescent polyfluorene," Appl Phys Lett 73, 1565-1567 (1998).

27 A J Campbell, D D C Bradley, and H Antoniadis, "Dispersive electron transport in an electroluminescent polyfluorene copolymer measured by the current integration time-of-flight method," Appl Phys Lett $\mathbf{7 9}$, 2133-2135 (2001).

28 C Adachi, T Tsutsui, and S Saito, "Blue light-emitting organic electroluminescent devices," Appl Phys Lett 56, 799-801 (1990).

29 N C Greenham, S C Moratti, D D C Bradley, R H Friend, and A B Holmes, "Efficient light-emitting-diodes based on polymers with high electron-affinities," Nature 365, 628-630 (1993).

30 Y Shen, D B Jacobs, G G Malliaras, G Koley, M G Spencer, and A Ioannidis, "Modification of indium tin oxide for improved hole injection in organic light emitting diodes," Adv Mater 13, 1234-1238 (2001).

31 M G Mason, L S Hung, and C W Tang, S T Lee, K W Wong, and M Wang, "Characterization of treated indium-tin-oxide surfaces used in electroluminescent devices,”J Appl Phys 86, 1688-1692 (1999).

32 M Leadbeater, "Polymers shine the light," Oemagazine 2, 14-17 (2002).

33 J W T Walsh, Photometry (Constable and Company Ltd, London, 1953), p. 321.

34 S-J Lee, A Badano, and J Kanicki, "Monte Carlo modeling of the light transport in polymer light-emitting devices on plastic substrates," IEEE J Select Topics Quantum Electron 10, 37-44 (2004).

35 N C Greenham, R H Friend, and D D C Bradley, “Angular dependence of the emission from a conjugated polymer light-emitting diode: implications for efficiency calculations," Adv Mater 6, 491-494 (1994).

36 I D Parker, "Carrier tunneling and device characteristics in polymer light-emitting diodes,” J Appl Phys 75, 1656-1666 (1994).

37 P W M Blom, M J J de Jong, and J J M Vleggaar, "Electron and hole transport in poly (p-phenylene vinylene) devices," Appl Phys Lett 68, 3308-3310 (1996).

38 P E Burrows, Z Shen, V Bulovic, D M McCarty, S R Forrest, J A Cronin, and M E Thompson, "Relationship between electroluminescence and current transport in organic heterojunction light-emitting devices," J Appl Phys 79, 7991-8006 (1996).

39 V Bulovic and S R Forrest, "Polymeric and molecular organic light emitting devices: a comparison," Semiconductors and Semimetals $\mathbf{6 5}$, $1-26(2000)$.

40 J P Bender, B J Norris, and J F Wager, "OLED modeling via SPICE," http://eecs oregonstate edu/matdev/pub html.

41 Y Bonnassieux and D B Van, "OLED SPICE modeling in a passive matrix of pixels,” Proc IDRC, 30-33 (2003).

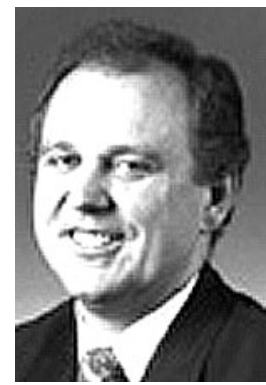

Jerzy Kanicki received his Ph.D. degree in sciences (D.Sc.) from the Free University of Brussels (ULB), Brussels, Belgium, in 1982. His dissertation research work involved the optical, electrical, and photovoltaic properties of undoped and doped trans-polyacetylene. He subsequently joined the IBM Thomas J. Watson Research Center, Yorktown Heights, New York, as a Research Staff Member working on hydrogenated amorphous silicon devices for photovoltaic and flatpanel-display applications. In 1994, he moved from the IBM Research Division to the University of Michigan as a Professor in the Department of Electrical Engineering and Computer Science. At the University of Michigan from 1994 to 2000, he did leading work on various flat-paneldisplay technologies. He started work in 2000 on variety of fundamental problems related to organic and molecular electronics. From 2002 to 2003, he spent a sabbatical year at the Center for Polymers and Organic Solids (Physics Department), University of California, Santa Barbara, conducting research in area of the conducting polymer devices. He is the author and coauthor of over 250 publications in journals and conference proceedings. He has edited two books and three conference proceedings. He is coauthor of the book High-Fidelity Medical Imaging Displays (Bellingham, Washington: SPIE Press, 2004). Dr. Kanicki has presented numerous invited talks at national and international meetings in area of organic and inorganic semiconductor devices.

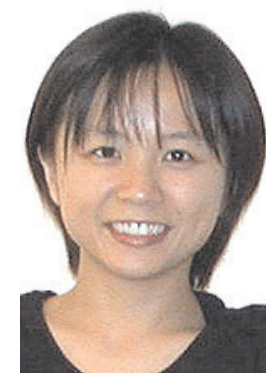

Dr. Shu-Jen Lee received her Ph.D. degree in macromolecular science and engineering at the University of Michigan, Ann Arbor in 2004. During her Ph.D. study, she joined the Organic and Molecular Electronics group in the electrical engineering department of the University of Michigan. Her interests are molecular design of the organic opto-electronics materials and optical design of organic light emitting devices. She is currently a senior process engineer at Intel.

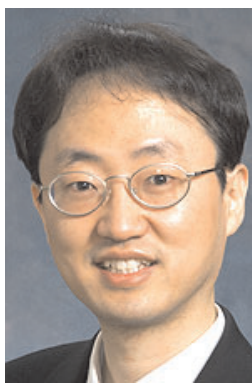

Yongtaek Hong received his B.S. and M.S. degrees in electronics engineering from Seoul National University, Seoul, South Korea, in 1994 and 1996, and his Ph.D. in electrical engineering from Univerity of Michigan, Ann Arbor, Michigan, USA, in 2003. Based on his Ph.D. research regarding a-Si:H TFT active-matrix polymer lightemitting displays, he has authored and co-authored more than 20 journal papers and conference presentations. He is currently with the Display Science \& Technology Center at Eastman Kodak Co., Rochester, New York, USA, as a senior research scientist. His research interests are low-cost backplane technologies for robust, flexible flat-panel displays and digital radiography sensor arrays. Dr. Hong received the Korea Foundation for Advanced Studies Scholarship from 1997 to 2002, and College of Engineering Graduate Student Distinguished Achievement Award from University of Michigan in 2003. He is a member of SID, IEEE, and SPIE. 